\title{
IMPACTO DE LA AMBULACIÓN TEMPRANA EN LA REDUCCIÓN DE CEFALEA Y DOLOR LUMBAR EN PACIENTES SOMETIDOS A ANESTESIA ESPINAL: REVISIÓN SISTEMÁTICA Y META-ANÁLISIS DE ESTUDIOS EXPERIMENTALES
}

\author{
IMPACT OF EARLY AMBULATION ON THE REDUCTION OF \\ HEADACHE AND BACK PAIN AFTER SPINAL ANAESTHESIA: A \\ SYSTEMATIC REVIEW AND META-ANALYSIS OF EXPERIMENTAL \\ STUDIES
}

\author{
Olga L. Cortés * \\ Maribel CANTOR ${ }^{* *}$ \\ Luz A. Mantilla *** \\ Diana M. Betancourt ${ }^{* * *}$
}

\begin{abstract}
RESUMEN
Objetivo: Determinar el impacto de la ambulación temprana (AT) en la presencia de cefalea y dolor lumbar en pacientes sometidos a procedimientos quirúrgicos bajo anestesia espinal. Material y método: Revisión sistemática y meta-análisis que revisó las bases de datos MEDLINE, CINAHL online, HealthStar, EMBASE, el Cochrane Library Controlled Trials Registry, LILACS y consulta de expertos. Incluyó estudios experimentales aleatorizados en cualquier idioma comparando pacientes sometidos a anestesia subaracnoidea aleatorizados durante su hospitalización a un grupo de ambulación temprana (intervención) o a un grupo control/cuidado estandarizado (reposo cama). La elegibilidad y la calidad de los artículos fue realizada por cuatro revisores de manera independiente. Luego se realizó la extracción de la información. Se calculó riesgo relativo (RR), 95 \% de intervalos de confianza usando el modelo de efecto random. Resultados: Respecto de la cefalea y dolor lumbar $24 \mathrm{~h}$ posteriores a la anestesia espinal se identificaron seis estudios entre 1988-2008, teniendo en cuenta los criterios de elegibilidad. Se observaron 41 cefaleas (16.9\% de 242$)$ y 24 dolor lumbar (21.6\% de 111$)$ entre pacientes asignados a una ambulación temprana (DT), comparado con 54 cefaleas ( $22.3 \%$ de 207$)$ y 32 dolor lumbar ( $27.5 \%$ de 116$)$ entre pacientes con anestesia espinal asignados al grupo control $(\mathrm{RR}=0.67,95 \% \mathrm{CI} 0.44,1.02 ; \mathrm{y}$ $\mathrm{RR}=0.79$, IC 95\% 0.46, 1.34 respectivamente). Conclusión: El meta-análisis sugiere que puede existir beneficio hacia una disminución de cefalea y dolor lumbar en pacientes que reciben AT posterior a anestesia espinal.
\end{abstract}

Palabras clave: Ambulación precoz, anestesia espinal, anestesia subaracnoidea, cuidado posoperatorio, metaanálisis.

\footnotetext{
ABSTRACT

Objective: To determine the impact of early ambulation (EA) versus late ambulation on total headache and back pain after spinal anaesthesia. Design: Systematic review and meta-analysis, used data Sources such as MEDLINE, CINAHL online, HealthStar, EMBASE, the Cochrane Library Controlled Trials Registry, LILACS and experts. Methods: Published randomized controlled trials in all languages comparing spinal anesthesia patients allocated to any in-hospital early ambulation or a control/standard treatment (bed rest). Four reviewers

* Enfermera. Docente Escuela de Enfermería; Grupo Investigación GRINFER. Universidad Industrial de Santander, Bucaramanga, Colombia.E-mail: olucof@uis.edu.co u olgacortesf@gmail.com

** Enfermera Hospital San Vicente de Arauca, Arauca, Colombia. E-mail: marvycan@hotmail.com

${ }^{* * *}$ Enfermera Clínica Carlos Ardila Lulle, Bucaramanga, Colombia. E-mail: lamr_0526@hotmail.com

${ }^{* * * *}$ Enfermera Fundación Cardio-Infantil, Bogotá, Colombia. E-mail: diamar45@hotmail.com
} 
independently assessed study eligibility and quality; and also performed data extraction. We calculated relative risks (RRs) and 95\% confidence intervals (CIs) using the random-effects model. Outcomes: cefalea or back pain $24 \mathrm{~h}$ post-spinal anaesthesia. Results: Six studies met our eligibility criteria published between 1988-2008. There were 41 cefalea events (16.9\% of 242) and 24 back pain events (21.6\% of 111) among patients receiving EM, compared with 54 cefalea events ( $22.3 \%$ of 207$)$ and 32 back pain events (27.5\% of 116) among spinal patients receiving control treatment (for cefalea $\mathrm{RR}=0.67,95 \% \mathrm{CI} 0.44,1.02$; and for lumbar pain $\mathrm{RR}=0.79$, IC $95 \%$ $0.46,1.34)$. Conclusion: Our meta-analysis suggests that there is a possible benefit towards decreased cefalea and back pain with EA after spinal anaesthesia.

Key words: Early ambulation, spinal anaesthesia, subarachnoid anaesthesia, posoperative care, meta-analysis.

Fecha recepción: 03/11/09 Fecha aceptación: 04/08/10

\section{INTRODUCCIÓN}

La anestesia regional se clasifica comúnmente como anestesia espinal y anestesia epidural (1). La anestesia se obtiene mediante la administración de anestésicos locales en el espacio subaracnoideo (anestesia espinal) o mediante la administración de anestésicos en el espacio epidural que rodea el saco de fluido espinal (1). Las soluciones anestésicas usadas para anestesia raquídea o espinal pueden ser hipobáricas o ligeras, con una densidad menor a la del LCR; hiperbáricas o pesadas, las cuales son más densas que el LCR; o isobáricas, aquellas que tienen igual peso específico que éste. Las soluciones hiperbáricas son usadas con frecuencia, y las presentaciones disponibles actualmente son la lidocaína pesada al 5\% y la bupivacaína al $0.5 \%$. Las hipobáricas requieren grandes volúmenes de anestésico y no se utilizan regularmente en la anestesia raquídea (2).

Las molestias transitorias que se generan por la administración de la anestesia subaracnoidea, tales como dolor lumbar y cefalea, han sido relacionadas con el trauma durante la aplicación de la anestesia con lesiones ligamentosas o del disco intervertebral, o relacionadas con el tipo de solución (e.g., hiperbáricas) utilizadas para este fin (3-5). El síndrome de dolor lumbar transitorio (DLT) se irradia a glúteos y miembros inferiores y puede tener una duración de más o menos
72 h (6). La cefalea posterior a la punción se presenta en el 25-60\% de los pacientes y puede persistir por una o varias semanas ( 7 , 8). La elevación de la cabeza del paciente al inicio de la ambulación temprana se ha determinado como causal del inicio de la cefalea fronto-occipital intenso, el cual puede disminuir con la posición supina $(6,9)$. Ocasionalmente esta cefalea se acompaña de tinitus, diplopía, náuseas y vómito (10). Sin embargo no existe una clara evidencia de la relación existente entre movilización y dolor lumbar y/o cefalea (3). La incertidumbre existente acerca de los patrones de ambulación posterior a la anestesia espinal, y la falta de guías de manejo basadas en la evidencia para esta intervención temprana, dificulta la toma de decisiones de enfermería durante la recuperación posanestésica. Esto se constituye en una necesidad que debe ser resuelta con el fin de proporcionar un cuidado oportuno, mejorar la calidad del cuidado a los usuarios y reducir los costos institucionales por complicaciones $(5,8,11)$. Con el fin de explorar acerca de esta incertidumbre relacionada con el cuidado de enfermería realizamos una revisión sistemática de la literatura y metaanálisis para analizar la siguiente pregunta: ¿Cuál es el impacto de la movilización temprana en la presencia de efectos como cefalea y dolor lumbar en pacientes sometidos a procedimientos quirúrgicos bajo anestesia espinal? 


\section{MATERIAL Y MÉTODO}

Este proyecto incluyó estudios en los cuales los individuos sometidos a anestesia espinal hubiesen sido aleatorizados a un grupo intervención y otros aleatorizados a un grupo control. La intervención experimental, aleatorizada, controlada se definió como ambulación temprana (AT), comparada con un grupo control, el cual podría estar expuesto a reposo en cama, movilización tardía, o cuidado convencional posterior a la anestesia. La movilización temprana debía incluir algún cambio desde la posición supina a la silla y/o el inicio de la deambulación después de la recuperación de la motricidad y sensibilidad de los miembros inferiores. Incluimos estudios experimentales reportados en cualquier lenguaje, sin tener en cuenta tiempo de publicación, o estado de la publicación; participantes adultos ( $>18$ años $\mathrm{y}<80$ años). Finalmente los estudios debían tener como resultado o evento de salida la evaluación de cefalea o dolor lumbar durante las primeras $24 \mathrm{~h}$ posteriores a la anestesia espinal.

Se dirigió una búsqueda de los estudios elegibles en bases de datos como MEDLINE (1980-2008), CINAHL online (1980-2008), HealthStar (1980-2008), LILACS (19802001), EMBASE (1980-2008), y el Cochrane Library Controlled Trials Registry (19802008), combinando términos de búsqueda de la población (adultos, anestesia subaracnoidea, espinal); una intervención (ambulación temprana, ambulación, caminar); y el método (estudio experimental, aleatorizado y controlado). Tres investigadores (LM, DB, MC) obtuvieron todos los títulos e identificaron los artículos que incluían los criterios relevantes para el estudio. Los tres investigadores leyeron inicialmente los métodos de los artículos con el fin de determinar su completa elegibilidad. Se realizaron estrategias adicionales con el fin de no excluir algún artículo relevante como la búsqueda manual de contenidos en revistas de anestesia; revi- sión de la bibliografía de la lista de artículos seleccionados; y consulta a anestesiólogos y enfermeras de salas de recuperación. Se realizó un análisis de elegibilidad en caso de ser identificados más estudios.

La búsqueda identificó 200 citaciones o artículos. Tres evaluadores (MC, LM, DB) de manera independiente evaluaron los títulos y resúmenes de cada artículo e identificaron todas las citaciones conteniendo los estudios de interés relacionadas con anestesia espinal o subaracnoidea y ambulación temprana. Todos los resúmenes identificados al menos por un revisor fueron seleccionados, y cada artículo fue evaluado en su totalidad, acorde con un formato de extracción de la información. Un cuarto evaluador (OC) leyó todos los documentos y extrajo la información de manera individual de cada artículo utilizando el mismo instrumento con el fin de validar los estudios seleccionados. La calidad de los datos fue evaluada de manera independiente por cada investigador teniendo en cuenta la escala de Jadad, la cual incluye el reporte de la aleatorización, el método de la aleatorización, el reporte del encubrimiento de la intervención (ciego), el seguimiento, y el retiro o pérdida de la población de estudio (12). La evaluación del acuerdo entre los 4 revisores de los artículos se realizó mediante el método Kappa, el cual se aplica con el fin de medir el acuerdo corregido del azar $(13,14,15)$. El análisis del acuerdo se realizó para la selección de los artículos teniendo en cuenta el título, el resumen, los métodos y la identificación de los resultados de los estudios (dolor lumbar y cefalea) $(13,16,17)$. El análisis de los estudios siguió el principio de intención (Intention to treat analysis), de tal forma que se incluyeron en el análisis todos los pacientes que habían sido aleatorizados inicialmente sirviendo como denominador.

Se realizó una sumatoria de las diferencias encontradas en cada uno de los estudios (efectos adversos), y se estimó el tamaño del efecto por medio de la identificación del riesgo relativo (RR) de cada uno de los estudios, 
y los intervalos de confianza del 95\% para cada evento de salida o resultado, utilizando el modelo de efectos aleatorios basados en el fundamento de que este modelo, a diferencia del modelo de efectos fijos, ofrece una varianza del efecto que incluye la varianza interestudio $(14,15,18)$. Este modelo asume que los estudios experimentales incluidos en esta revisión representan una muestra aleatoria de todos los estudios experimentales que son potencialmente disponibles en el presente y en el futuro. Así mismo se analizó la heterogeneidad de la medida del efecto utilizando la prueba estadística chi cuadrado. Se evaluaron datos en duplicado y todos los cálculos se realizaron utilizando Review Manager version 4.2 (The Cochrane Colla- boration, Oxford, UK).

\section{RESULTADOS}

El análisis Kappa (acuerdo corregido del chance) para la elegibilidad de los artículos teniendo en cuenta los criterios previamente establecidos fue de 0.8 (95\% CI 0.7-0.9). Este proceso de búsqueda condujo a la identificación de 11 publicaciones, de las cuales sólo 6 fueron incluidas para esta revisión teniendo en cuenta los criterios de selección $(8,19-23)$; y 5 fueron excluidas $(4,24-27)$ (Tabla 1$)(\mathrm{Fi}-$ gura 1). La razón de exclusión de los estudios se relaciona con la identificación de eventos

Tabla 1. Características de los Estudios Excluidos.

\begin{tabular}{|l|c|}
\hline Autor, año, país & Motivo de Exclusión \\
\hline Collis, 1999, Inglaterra & Estudia al feto y la gestante \\
\hline Imarengiaye, 2003, Canadá & Estudia sensibilidad \\
\hline Watsson, 2003, Canadá & Compara 2 tipos de medicamentos \\
\hline Tong, 2003, Canadá & Compara 2 intervenciones con lidocaína 1 y $5 \%$ \\
\hline Golara, 2002, Inglaterra & Dilatación, expulsivo y trauma perineal \\
\hline
\end{tabular}

de salida diferentes al objeto de nuestro estudio (cefalea o dolor lumbar), tales como dilatación del cuello (trabajo de parto), expulsivo, estado del feto y la gestante; tener una intervención diferente a la movilización temprana (comparación de fármacos anestésicos, comparación de agujas) o punción espinal con objetivo diagnóstico (con medio de contraste diagnóstico).

Se incluyeron estudios de pacientes sometidos a cirugías como procedimientos ortopédicos de extremidades inferiores incluyendo cadera, cirugías rectales, resecciones abdomino-perineales, cirugías pélvicas y abdominales; cirugías obstétricas incluyendo cesáreas; cirugías del tracto genitourinario; resecciones transuretrales, ci- rugía de la vejiga, cirugía vascular de pelvis y de miembros inferiores, manejo del dolor oncológico o no oncológico (epidurales) y cirugía inguinal.

Una vez seleccionados los artículos, se extrajo la información encontrándose que los 6 artículos fueron realizados en diferentes países como Holanda, Suecia, Australia, USA, Finlandia y Grecia, escritos en inglés. La población total fue de 565 pacientes (18 pacientes no siguieron el estudio y 9 se perdieron, sin más referencias), con edades comprendidas entre 21 y 68 años. Todos los estudios incluyeron ambos sexos $(8,19,21,22)$, excepto dos que incluyeron un solo género como el estudio de Thornberry (23), el cual incluyó sólo mujeres (parto o alumbramiento) y el 


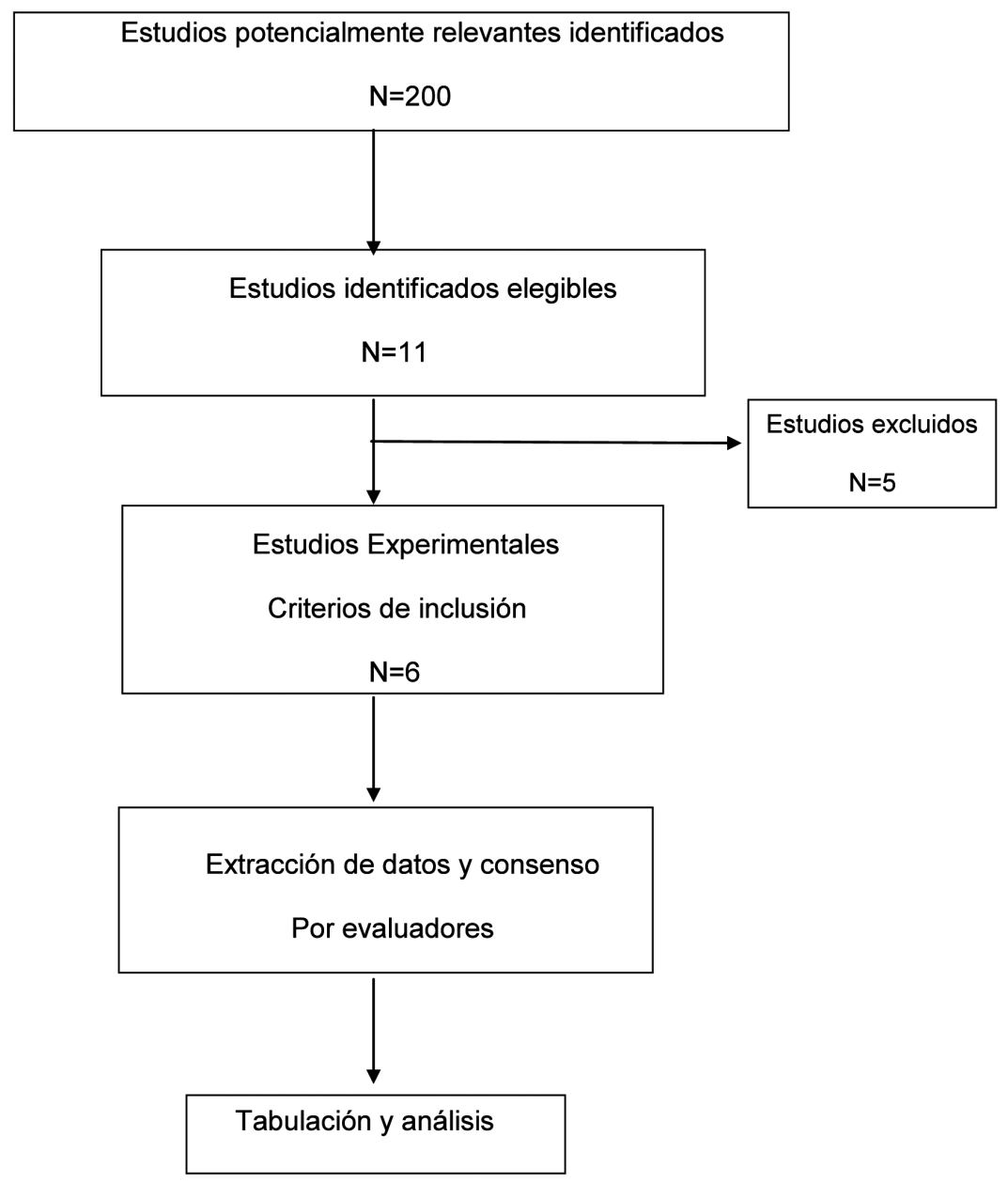

Figura 1. Flujograma de selección de los estudios.

estudio de Fassoulaki (20) que incluyó sólo hombres (resección de próstata). Los procedimientos estudiados con anestesia espinal fueron herniorrafias, cirugías urológicas menores, artroscopias y resecciones transuretrales. Tres estudios fueron realizados antes del año 2000 (1988-1991) y otros tres fueron realizados entre el año 2001 y 2005; el tipo de anestesia usada en los estudios fue la lidocaína al 2\% hiperbárica; el 50\% de los estudios utilizó aguja para administrar la anestesia $\mathrm{n}^{\circ}$ 26; y el $33.3 \%$ de los estudios utilizó agujas $\mathrm{n}^{\circ} 22, \mathrm{n}^{\circ} 25 \mathrm{y} \mathrm{n}^{\circ} 27$. El tiempo de la movilización temprana posterior a la anestesia subaracnoidea y la recuperación de la motricidad y sensibilidad de miembros inferiores se observó en los estudios en un intervalo entre
2 h 30 min y 6 h . La movilización tardía o reposo en cama se observó después de $24 \mathrm{~h}$ pospunción lumbar (Tabla 2).

Los seis estudios fueron experimentales, en los cuales los pacientes fueron aleatorizados por algún método a un grupo de movilización temprana (intervención) o a un grupo de reposo absoluto o control. Sin embargo sólo dos estudios reportaron los números aleatorios en sobres como método para la aleatorización Silvanto (22) y Thornberry (23). En todos los estudios el seguimiento fue mayor del $90 \%$ y se reporta el número de pacientes que desertó del estudio como en el estudio de Cook (19). Sin embargo, aunque todos los estudios reportan haber utilizado el encubrimiento (ciego) simple de la interven- 
Tabla 2. Características de los Estudios Incluidos.

\begin{tabular}{|c|c|c|c|c|c|c|}
\hline \multirow{2}{*}{$\begin{array}{l}\text { Autor, } \\
\text { año, país }\end{array}$} & \multirow[b]{2}{*}{ Sexo } & \multirow{2}{*}{$\begin{array}{c}\text { Tipo de } \\
\text { procedimiento }\end{array}$} & \multirow{2}{*}{$\begin{array}{l}\text { Protocolo de } \\
\text { movilización según } \\
\text { valoración+ } \\
\text { Grupo intervención }\end{array}$} & \multicolumn{2}{|c|}{ Hora la movilización } & \multirow{2}{*}{$\begin{array}{c}\text { Resultados } \\
\text { Calibre aguja } \\
\#\end{array}$} \\
\hline & & & & Actividad & Control & \\
\hline $\begin{array}{l}\text { Cramer, 2005, } \\
\text { Holanda }\end{array}$ & $\begin{array}{l}{ }^{\star} \mathrm{F}: 19 \\
{ }^{*} \mathrm{M}: 41\end{array}$ & Cirugía menor & $\begin{array}{l}\text { Levantarse y } \\
\text { caminar } 2 \mathrm{~h}\end{array}$ & $<6 \mathrm{~h}$ & $>6 \mathrm{~h}$ & 27 \\
\hline $\begin{array}{l}\text { Lindh, 2001, } \\
\text { Suecia }\end{array}$ & $\begin{array}{c}\mathrm{F}: 2 \\
\mathrm{M}: 105\end{array}$ & $\begin{array}{c}\text { Reparo de } \\
\text { hernia inguinal }\end{array}$ & $\begin{array}{l}\text { Levantarse y } \\
\text { caminar } 5 \mathrm{~h}\end{array}$ & $5 \mathrm{~h}$ & $12 \mathrm{~h}$ & 25 у 26 \\
\hline $\begin{array}{l}\text { Cook,1989, } \\
\text { Australia }\end{array}$ & $\begin{array}{l}{ }^{* *} \mathrm{~F}: 28 \\
\mathrm{M}: 74\end{array}$ & $\begin{array}{c}\text { Urológicas } \\
\text { Ginecológicas }\end{array}$ & $\begin{array}{l}\text { Levantarse y } \\
\text { caminar } 4 \mathrm{~h}\end{array}$ & $4 \mathrm{~h}$ & $24 \mathrm{~h}$ & 22 \\
\hline $\begin{array}{l}\text { Thornberry, } \\
\text { 1988, U.S.A. }\end{array}$ & F: 80 & Posparto & $\begin{array}{l}\text { Levantarse y } \\
\text { Caminar } 6 \mathrm{~h}\end{array}$ & $<6 \mathrm{~h}$ & $24 \mathrm{~h}$ & 25 у 26 \\
\hline \begin{tabular}{|l} 
Silvanto, 2004, \\
Finlandia \\
\end{tabular} & $\begin{array}{c}\mathrm{F}: 4 \\
\mathrm{M}: 116\end{array}$ & $\begin{array}{l}\text { Artroscopia } \\
\text { de rodilla }\end{array}$ & $\begin{array}{l}\text { Levantarse y } \\
\text { caminar } 3 \mathrm{~h}\end{array}$ & $<6 \mathrm{~h}$ & $18-24 \mathrm{~h}$ & 26 у 27 \\
\hline $\begin{array}{l}\text { Fassoulaki, } \\
\text { 1991, Grecia }\end{array}$ & M:69 & $\begin{array}{c}\text { Resección próstata } \\
\text { transuretral }\end{array}$ & $\begin{array}{l}\text { Levantarse y } \\
\text { caminar } 8 \mathrm{~h}\end{array}$ & $6-8 \mathrm{~h}$ & $24 \mathrm{~h}$ & 22 \\
\hline
\end{tabular}

${ }^{*}$ F: Femenino ${ }^{\star}$ M: Masculino; ${ }^{* *} 18$ Pacientes no siguieron en el estudio. + Movilización grupo intervención se inició acorde con la valoración y recuperación de sensibilidad, temperatura y motricidad posterior a la anestesia espinal.

ción, sólo los de Cook (19) y Fassoulaki (20) fue claramente encubierto (proveedor de sareportan el personal dentro del estudio que lud en sala de recuperación) (Tabla 3).

Tabla 3. Calidad de los Estudios.

\begin{tabular}{|l|c|c|c|c|}
\hline Autor /año & Realizada & $\begin{array}{c}\text { Método de aleatorización } \\
\text { reportado }\end{array}$ & $\begin{array}{c}\text { Encubrimiento } \\
\text { reportado }\end{array}$ & $\begin{array}{c}\text { Seguimiento } \\
\mathbf{9 0 \%}\end{array}$ \\
\hline Cramer, 2005 & Sí & No & No & ND \\
\hline Lindh, 2001 & Sí & No & No & Sí \\
\hline Cook, 1989 & Sí & No & Sí & Sí \\
\hline Thornberry, 1988 & Sí & Números aleatorios en sobres & No & Sí \\
\hline Silvanto, 2004 & Sí & Números aleatorios en sobres & No & Sí \\
\hline Fassoulaki, 1991 & Sí & No & Sí & Sí \\
\hline
\end{tabular}

Criterios de evaluación de los estudios elaborado por Jadad A. 1996.

Del total de los estudios incluidos ( $\mathrm{n}=6)$, todos evaluaron cefalea, excepto en el realizado por Lindh (21). Sólo dos estudios evaluaron dolor lumbar y fueron incluidos en el análisis Cook (19) y Lindh (21). El total de eventos reportados en los estudios incluyó 105 cefaleas y 56 dolores lumbares. La gráfica de funnel plot que representa la talla del efecto para los estudios individuales presentó una aceptable simetría para los dos resultados y así se procedió a la realización de la suma del efecto de los resultados.

Se observaron 41 casos de cefalea (16.9\%) entre 242 pacientes que recibieron anestesia espinal aleatorizados a movilización temprana, comparado con 54 casos de cefalea 


\section{DISCUSIÓN}

El presente estudio indica que existe un gradiente de reducción de eventos como cefalea y dolor lumbar en pacientes sometidos a anestesia espinal con fines quirúrgicos que inician la ambulación tempranamente. Los datos presentados en este meta-análisis a partir de estudios experimentales exponen la necesidad de la realización de estudios experimentales con mayor población, con el fin de evaluar el efecto de la AT posterior a una anestesia espinal.

Los hallazgos de este estudio indican una tendencia hacia la reducción de la cefalea del $33 \%$ en pacientes que fueron tempranamente movilizados entre 5-6 h después de haber recuperado su sensibilidad y motricidad comparado con los pacientes que estuvieron en reposo en cama o en el cuidado convencional (RR 0.67, p=0.06). Aunque el efecto de la movilización temprana no fue significativo, la frecuencia de cefalea se observó más en el grupo de movilización tardía que en el grupo de deambulación temprana (54 vs 41 ). Los hallazgos encontrados sugieren que no existe evidencia obtenida de los estudios ECAs (experimentales aleatorizados, controlados) indicando que el reposo en cama como intervención ofrezca algún beneficio posterior a la anestesia espinal. Por el contrario se observa una tendencia hacia la disminución de la cefalea en pacientes que reciben anestesia espinal e iniciaron la caminata en el servicio tempranamente.

Los eventos para dolor lumbar también se observaron con mayor frecuencia en el grupo de movilización tardía, sin embargo sólo dos estudios incluyeron este evento final dentro de su análisis. Se observó una reducción del dolor lumbar del 21\% en los pacientes que fueron movilizados más tempranamente comparado con los que estuvieron en reposo en cama $(R R 0.79, p=0.4)$. Este efecto puede ser sobre estimado pues la muestra de pacientes fue pequeña y sólo dos estudios re- visaron este evento (111 pacientes intervención, 116 pacientes cuidado estándar).

Poca evidencia existe acerca del beneficio de una intervención como la movilización temprana a pesar de que prescribe de forma rutinaria. La evidencia reportada en la literatura científica nos presenta dos revisiones sistemáticas que exploraron los efectos del reposo en cama y han estudiado directamente los efectos de la movilización temprana posterior a anestesia espinal o punción lumbar. El primer estudio fue realizado por Allen, Glasziou, Del Mar (28), una revisión sistemática de la literatura, la cual exploró los efectos de la prescripción de reposo prolongado en cama comparado con la movilización temprana de pacientes con diferentes condiciones como dolor lumbar, parto espontáneo, infarto agudo del miocardio, cateterismo cardiaco y anestesia espinal entre otros. Los participantes recibieron el mismo tratamiento excepto el tiempo de reposo en cama. Sin embargo el estudio incluyó pocos pacientes sometidos a anestesia espinal (4 casos), tres de los cuales fueron incluidos en el nuestro por ser considerado un estudio aleatorizado y controlado, ECA $(19,20,23)$. En general este estudio no asumió eficacia con el reposo en cama al encontrar un aumento de efectos negativos tales como cefalea, náuseas y vómito en pacientes, quienes recibieron esta prescripción, similar a nuestros hallazgos.

El segundo estudio desarrollado por Sudlow y Warlow (3), una revisión sistemática de la literatura y meta-análisis, exploró los cambios de posición y la administración de fluidos para la prevención de cefalea pospunción dural diagnóstica. Este incluyó estudios aleatorizados controlados (ECAs) (3). Este estudio fue muy similar al nuestro, con la diferencia que en éste se incluyeron pocos estudios de pacientes sometidos a anestesia espinal para la realización de algún procedimiento quirúrgico, y mayor número de estudios relacionados con procedimientos con fines diagnósticos como punción lumbar diagnóstica, mielografía, administración in- 
tratecal de drogas, y punción accidental de la duramadre en anestesia epidural. Catorce estudios fueron incluidos y sólo tres se relacionaron con la administración de anestesia espinal y la movilización temprana versus tardía, dos de los cuales fueron incluidos en el nuestro $(19,20)$. Los otros estudios incluyeron intervenciones como punción lumbar y administración de líquidos posterior a la punción diagnóstica. Los resultados demostraron que no existe diferencia entre movilización temprana versus tardía en la aparición de cefalea posterior a punción dural. Sin embargo este estudio hizo énfasis en la punción espinal con fines diagnósticos y no con fines anestésicos, no identificó otros estudios relacionados con anestesia espinal y movilización temprana disminuyendo la población del estudio y así disminuyendo la precisión en las inferencias obtenidas (3).

En general estos resultados, aunque son el producto de un trabajo de selección rígido, deben ser interpretados de manera conservativa. Es posible obtener una indicación del grado de incertidumbre del beneficio potencial de un tratamiento mediante el cálculo de los intervalos de confianza. Así mismo los estudios experimentales evitan el sesgo cuando incluyen métodos apropiados como una aleatorización apropiada de pacientes a los grupos de estudio, y cuando pacientes, investigadores y asesores están encubiertos. Los estudios que fallan en la inclusión de estas precauciones o criterios de calidad muestran una tendencia a reportar estimados de mayor amplitud del efecto de una intervención (17). Todos los estudios que nosotros incluimos fueron aleatorizados y controlados, y se incluyeron todos los reportados motivo de estudio. El método de aleatorización y tipo de persona en el proceso de investigación que estaba encubierto no fue reportado por todos los estudios, excepto Cook (19) y Fassoulaki (20).

Aunque se observaron diferencias en el año de la realización de los estudios, el más antiguo fue realizado en 1988 y el más ac- tualizado fue realizado en 2005, se estima que existen pocas variaciones fundamentales relacionadas con la técnica de anestesia espinal, o relacionadas con el tipo de medicamento, calibre de la aguja, lo cual indica que hay homogeneidad en el procedimiento hasta nuestros días. El rango de edad de los pacientes fue de 21 a 68 años, y no se presentó mortalidad en los pacientes estudiados.

En general nuestro trabajo produce un impacto positivo en el cuidado de enfermería, especialmente para aquellos profesionales que laboran en el servicio de cirugía y recuperación posanestesia con una tendencia hacia la no eficacia del reposo en cama, procedimiento o intervención que es aún utilizado como rutina para el cuidado de estos pacientes. Este estudio permite tener un grado menor de incertidumbre en el cuidado de enfermería al momento de realizar la movilización temprana en pacientes que han tenido una anestesia espinal. La cautelosa implementación de la movilización temprana debe ser realizada teniendo en cuenta una valoración adecuada de la motricidad y sensibilidad de los miembros inferiores posterior a recibir la anestesia espinal; así como la realización de una evaluación del estado de salud del individuo de manera constante (parámetros hemodinámicos y un seguimiento de varias horas posterior a la anestesia espinal). Es importante la realización de más estudios experimentales aleatorizados controlados, incluyendo una mayor muestra de población con el fin de investigar no sólo los beneficios de la movilización temprana a la luz de los nuevos anestésicos, sino de verificar mayor poder en los resultados que permitan trasformar el cuidado. Es importante avanzar en la formulación de evidencia válida en el cuidado directo clínico en enfermería, y en este caso acorde con el progreso de la ciencia en los procedimientos anestésicos y medicamentos.

Los profesionales de enfermería deben evaluar el impacto que tiene potencialmen- 
te la ambulación temprana en la calidad de vida de los individuos, en la reducción de costos hospitalarios relacionados con tiempo de estadía y reducción de las complicaciones como cefalea y dolor lumbar. De esta forma avanzaremos más hacia una práctica basada en la evidencia en enfermería.

Este estudio de características únicas en relación a sus objetivos, y sus implicaciones para la práctica (evidencia tipo IA) sobre la movilización temprana de pacientes sometidos a anestesia espinal, una práctica que compete a enfermería, facilita la toma de decisiones en el cuidado directo y abre una oportunidad para la realización de futuros estudios de enfermería experimentales que fortalezcan nuestro propio conocimiento.

\section{REFERENCIAS}

1. Rodgers A, Walter N, Schung S, McKee A, Kehlet H, Zundert D, et al. Reduction of postoperative mortality and morbidity with epidural or spinal anaesthesia: results from overview of randomized trials. BMJ. 2000; 321(7275): 1-12.

2. Schneider M, Ettlin T, Kaufmann M, Schumacher P, Urwyler A, Hamp P.K., et al. Transient neurologic toxicity after hyperbaric subarachnoid anesthesia with 5\% lidocaine. Anesth Analg. 1993; 76 (5): 1154-1157.

3. Sudlow C, Warlow C. Posture and fluids for preventing post-dural puncture headache. $2^{\text {nd }}$ ed. Oxford: The Cochrane Library, Copyright; 2008.

4. Tong D, Wong J, Chung F, Friedlander M, Bremang J, Mezei G, et al. Prospective study on incidence and functional impact of transient neurologic symptoms associated with $1 \%$ versus $5 \%$ hyperbaric lidocaine in short urologic procedures. Anesthesiology. 2003; 98 (2): 485-494.

5. Whizar VM, Martínez N. Polémicas en Anestesia Subaracnoidea. Anestesia en
México [revista en internet] 2004 abriljunio. Hallado en: http://www.anestesiaenmexico.org/RAM2/controversias/ controversias.htm. [acceso el 5 septiembre de 2007].

6. García MJ, Meléndez HJ. Náusea y dolor en cesárea. Incidencia de náusea y dolor transoperatorio bajo diferentes técnicas de anestesia regional en cesárea. Rev Col Anest. 2003; 31(1): 9-19.

7. Aguilera-Castro F. Manejo de la cefalea post-punción accidental de la duramadre. Rev Col Anest. 1995; 23(3): 313-320.

8. Cramer BG, Stienstra R, Dahan A, Arbous MS, Veering BTh, Van Kleef JW. Transcient neurological symptom with subarachnoid lidocaine: Effect of early mobilization. Eur J Anaesthesiol. 2005; 22(1): 35-39.

9. Hampl KF, Schneider MC, Ummenhofer W, Drewe J. Transient neurologic symptoms after spinal anesthesia. Anesth Analg. 1995; 81(6): 1148-1153.

10. Spelina KR, Farcs HR, \& Gerber MD. Nausea and vomiting during spinal anaesthesia. Anaesthesia; 1984; 39 (2): 132-137.

11. Cajina RE. Cefalea post-punción dural en pacientes de cirugía electivas sometidos a bloqueo espinal con agujas número 25 G, 26 G y 27 G del Hospital Escuela Oscar Danilo Rosales Argüello, enerodiciembre 2004 [tesis de Grado Especialista en Anestesiología]. Universidad Nacional Autónoma de Nicaragua; 2005.

12. Jadad AR, Moore RA, Carroll D, Jenkinson C, Reynolds DJ, Gavaghan DJ, et al. Assessing the quality of reports of randomized clinical trials: is blinding necessary? Control Clin Trials. 1996; 17(1): 1-12.

13. Donner A, Klar N. The statistical analysis of Kappa statistics in multiple Samples. J Clin Epidemiol; 1996; 49(9): 1053-1058.

14. Oxman AD, Cook DJ, Guyatt GH. Users'guides to the medical literature. VI. How to use an overview. Evidence- 
Based Medicine Working Group. JAMA. 1994; 272(17): 1367-1371.

15. Oxman AD, \& Guyatt GH. A consumer's guide to subgroup analyses. Ann.Intern. Med. 1992; 116(1):78-84.

16. Fleiss JL. Measuring agreement between two judges on the presence or absence of a trait. Biometrics. 1975; 31(3): 651-659.

17. Le Lorier J, Gregoire G, Benhaddad A, LaPierre J, Derderian F. Discrepancies between meta-analysis and subsequent large randomized, controlled trials. N Engl J Med. 1997; 337(8): 536-542.

18. Der Simonian R, Laird N. Meta-analysis in clinical trials. Cont Clin Trial. 1986; 7(3): 177-188.

19. Cook PT, Davies MJ, Beavis RE. Bed rest and postlumbar puncture headache the effectiveness of 24 hours' recumbency in reducing the incidence of postlumbar puncture headache. Anaesthesia. 1989; 44(5): 389-391.

20. Fassoulaki A, Sarantopoulos C, Andreopoulou K. Early mobilization associated with lower incidence of post spinal headache? A controlled trial in 69 urologic patients. Anaesthesiol Reanim. $1991 ; 16$ (6): 375-378.

21. Lindh A, Anderson AS, Westman L. Is transient lumbar pain after spinal anaesthesia with lidocaine influenced by early mobilisation? Acta Anaesthesiol Scand. 2001; 45(3): 290-293.

22. Silvanto M, Tarkkila P, Mäkelä ML, Ro- senberg PH. The influence of ambulation time on the incidence of transient neurologic symptoms after lidocaine spinal anaesthesia. Anesth Analg. 2004; 98(3): 642-646.

23. Thornberry EA, Thomas TA. Posture and post-spinal headache a controlled trial in 80 obstetric patients. $\mathrm{Br} \mathrm{J}$ Anaesth. 1988; 60(2): 195-197.

24. Collins RE, Harding SA, Morgan BM. Effect of maternal ambulation on labour with low-dose combined spinal -epidural analgesia. Anaesthesia. 1999; 54(6): 535539.

25. Charles O, Song D, Prabhu A, Chung F. Spinal anesthesia: Functional balance is impaired after clinical recovery. Anesthesiology. 2003; 98 (2): 511-515.

26. Golara M, Plaat F, Shenan AH. Upright versus recumbent position in the second stage of labour in women with combined spinal-epidural analgesia. Int J Obstet Anesth. 2002; 11(1): 19-22.

27. Watson MW, Mitra D, Mclintock TC, Grant SA. Continuous versus singleinjection lumbar plexus blocks: Comparison of the effects on morphine use and early recovery after total knee arthroplasty. Reg Anesth Pain Med. 2005; 30 (6): 541-547.

28. Allen C, Glasziou P, Del Mar C. Bed rest: a potentially harmful treatment needing more careful evaluation. The Lancet. 1999; 354(9186): 1229-1233. 